\title{
AIRGLOW AND ITS IMPORTANCE ON MONITORING THE UPPER ATMOSPHERE: OBSERVATIONS AT COMMANDANT FERRAZ ANTARCTIC STATION
}

http://dx.doi.org/10.4322/apa.2014.092

\author{
José Valentin Bageston ${ }^{1}$, Paulo Prado Batista², Delano Gobbi², Hisao Takahashi², \\ Neusa Maria Paes-Leme ${ }^{2} \&$ Cristiano Max Wrasse ${ }^{2}$
}

'Universidade Tecnológica Federal do Paraná, Guarapuava-PR

2Instituto Nacional de Pesquisas Espaciais, São José dos Campos-SP, Rua Presidente Zacarias de Góes, 875, CEP 85015-430, Guarapuava, PR, Brazil

*e-mail: bageston@gmail.com

\begin{abstract}
The present paper presents and discusses the airglow phenomena and its importance on research activities related to the thermal structure and to the wave dynamics of the upper atmosphere at altitudes greater than $80 \mathrm{~km}$. We will focus on the basic definitions, instrumentation and examples of airglow data. First an introduction will be presented, followed by the Airglow and Aurora definitions. Also, we will present the instrumentation used to observe airglow emissions at the Brazilian Antarctic Station Commandant Ferraz (EACF) and atmospheric parameters/phenomena such as temperature and gravity waves. By using these observations we are able to study and understand the thermal structure and dynamics of the upper atmosphere. In the section of results we will present a time series of temperature and an example of wave event observed above Ferraz station, in order to illustrate the applicability of Airglow observations. In the discussion and conclusion session we emphasize the importance of maintain the upper atmosphere monitoring and other atmospheric observations at the Brazilian Antarctic Station.
\end{abstract}

Keywords: Airglow and Aurora, Mesosphere and Thermosphere, Temperature, Gravity Waves.

\section{Introduction}

The Airglow group (LUME) of the Aeronomy Division (DAE) of the National Institute for Space Research (INPE) uses optical equipment in order to study atmospheric phenomena related the physical and chemical processes that occur in the middle and high atmosphere (region above $80 \mathrm{~km}$ ). The LUME group has been operated optical equipments systematically at the Commandant Ferraz Antarctic Station (EACF) since 2002. Based in the evolution of the instrumentation used to monitor the upper atmosphere in the last decade, significant improvements have occurred in atmospheric research. In this sense, we should point out the installation of a latest generation Meteoric Radar at the Brazilian Antarctic Station in 2010 as a consequence of an international collaboration with the Project "Southern Andes-ANtarctic GRavity wave InitiAtive", sponsored by the National Science Foundation (NSF) of the
United Station of America. This new instrument is capable of several measurements, including the meteor flux in the upper atmosphere and mesospheric winds (Fritts et al., 2012). The operation of the meteor radar simultaneously with optical observations of the atmosphere will allow a better understanding of the wave's phenomena in the middle atmosphere and its connections with lower atmosphere, such as meteorological phenomenon and planetary waves. Gravity wave studies in Antarctica through the monitoring of airglow emissions have emerged in the last decade (Espy et al., 2004; Nielsen et al., 2006, 2009; Bageston et al., 2009, 2011a, b). Investigation of gravity wave sources, as observed in the mesosphere with airglow imager, over the King George Island and also above the Antarctica Peninsula is a current subject of study. In this type of study, winds and temperature data are used in combination with the

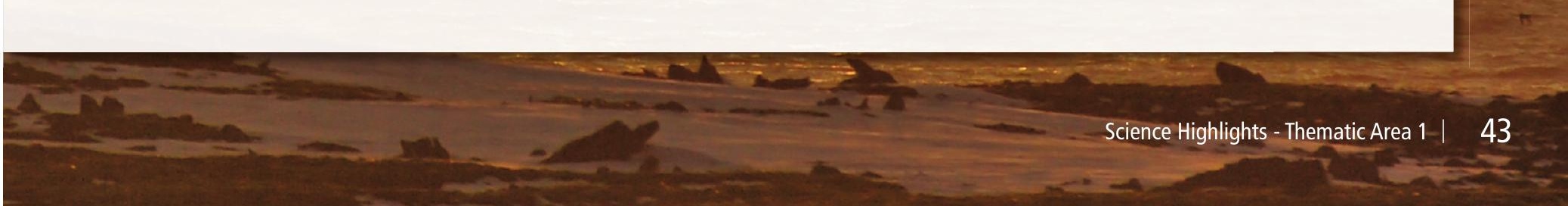


observed gravity wave parameters through airglow imagers and reanalysis data. Furthermore, images taken from meteorological satellites are used to correlate the origin of mesospheric waves to the meteorological phenomenon in troposphere. In the present article we will provide the basic definitions of the airglow and aurora phenomena. Also, it will be showed two important instruments (airglow cameras) used in the monitoring of the mesospheric temperature and in the observation of gravity wave above the Commandant Ferraz Station. Some examples of observed data will be shown in the result section.

\section{Materials and Methods}

\section{The Phenomena of Airglow and Aurora}

Airglow is an optical phenomenon of the high atmosphere which occurs both during the day and at night. These emissions of this tenuous light in the atmosphere originate directly or indirectly from solar radiation. The solar radiation leads atoms and molecules to excited states that when decay to their fundamental states of energy release the excess energy in the form of electromagnetic radiation. These airglow radiations are very thin lined emissions, which are very difficult to observe during the day because of the solar light contamination. However, during moonless nights these atmospheric radiations can be observed by equipments especially developed for this purpose. The airglow emissions occur in lines and bands spectrum and can be observed only through very sensitive optical equipment (CCD cameras) which makes use of interference filters that select specific lines or band emissions. The Sunlight spectrum and some Airglow emissions are shown in Figure 1, where discrete spectral lines and continuum spectrum bands are identified.
- Source: http://www.atoptics.co.uk/highsky/airglow2. htm.

Initially, the Airglow emissions were confused with the Aurora's lights (spectrum shown in Figure 2) because some spectral lines (atomic oxygen) observed in the Auroras are the same as those of Airglow. On the other hand, Auroras also show lines of molecular nitrogen $\left(\mathrm{N}_{2} \mathrm{e} \mathrm{N}_{2}^{+}\right)$.

- Source: http://www.atoptics.co.uk/highsky/auror3.htm.

Auroras occur in Polar Regions during the night and now is known that they have a very distinct origin of the Airglow emissions. The physical process of Aurora's origin is related to the excitation of atoms and molecules in the upper atmosphere from direct interaction with energetic particles (protons and electrons) originated from the Solar Wind. When these solar particles reach the top of the atmosphere near the Earth's poles, guided by the magnetic field lines, the interaction between these energetic particles and atmospheric constituents $\left(\mathrm{N}_{2}, \mathrm{~N}_{2}^{+}\right.$and $\left.\mathrm{O}\right)$ generates the Aurora phenomenon. Differently from the Aurora emissions, the Airglow is not caused by the collision of energetic particles with the atmospheric components, but has its origin associated to the solar radiation as already mentioned. The Airglow is characterized by occurring around the whole terrestrial globe, not restricting to specific regions of the planet, as is the case of the Auroras. One should observe that at high latitudes the Airglow emissions are strongly contaminated by the Aurora lights.

- The Airglow emissions as a tracer of the atmosphere

The emissions of Airglow have been used to monitor the atmosphere remotely. The main applications are related to the mesospheric temperature, wind and the dynamics of the Mesosphere/Thermosphere (Taylor et al., 1995; Smith et al., 2003; Medeiros et al., 2007; Bageston et al., 2007, 2009;

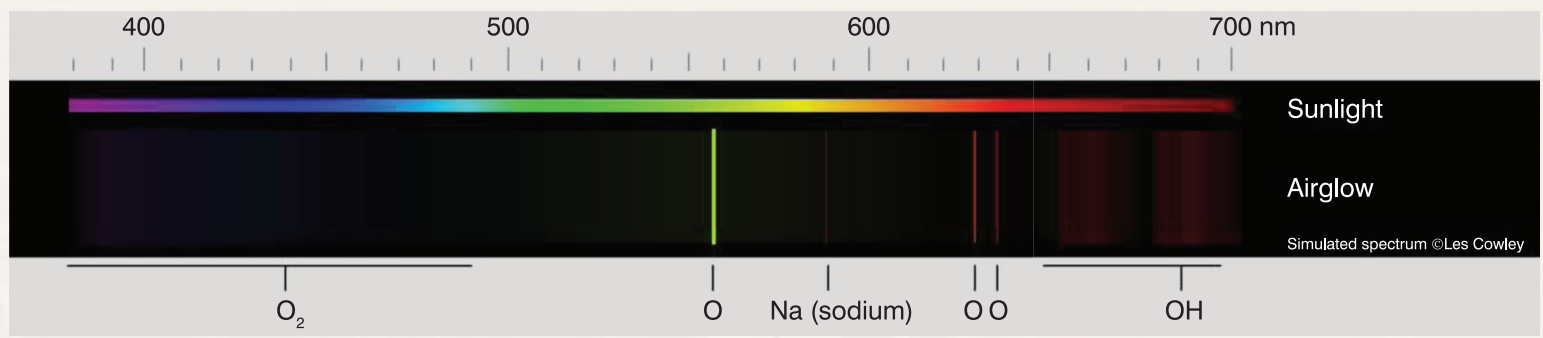

Figure 1. Sunlight and Airglow spectrum - The green line of oxygen is the strongest line of the Airglow and occurs mainly at heights between 90 and $100 \mathrm{~km}$, while the red line is a bit weaker and originates at altitudes higher than $200 \mathrm{~km}$. Sodium $(\mathrm{Na})$, Hydroxyl radicals $(\mathrm{OH})$, and oxygen molecules $\left(\mathrm{O}_{2}\right)$ complete the Airglow spectrum. 


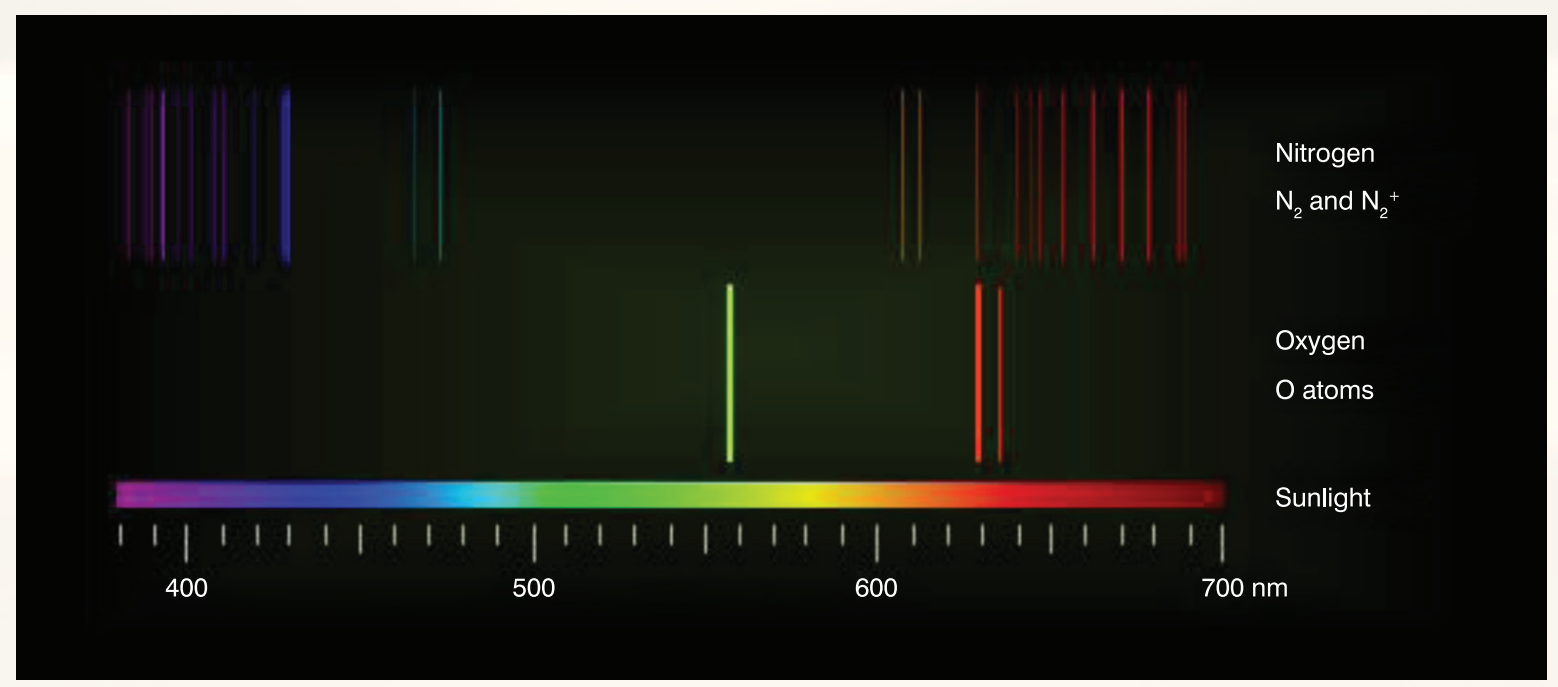

Figure 2. Aurora spectrum and continuous Sunlight - In the upper part are shown the main spectral lines associated to Nitrogen $\left(\mathrm{N}_{2}\right.$ and $\left.\mathrm{N}_{2}{ }^{+}\right)$and in the middle are presented to Oxygen lines. In the bottom part is the continuous Sunlight spectrum. In the bottom part is shown the continuous Sunlight spectrum. The Aurora emissions occur mainly at the Thermosphere.

Paulino et al., 2011; Amorim et al., 2011). The phenomenon of ionospheric bubbles (Equatorial plasma bubbles), which occurs in the Equatorial atmosphere and at low altitudes is of extreme interest as much from the point of view of basic science, as from the practical point of view, that is, regarding telecommunications and global positioning systems, such as GPS, since such phenomenon can significantly affect radio signals (Paulino et al., 2011 and references therein).

The observations of airglow at EACF initiated in 2002 with the installation of a Photometer to observe the emission of the band of hydroxyl, $\mathrm{OH}$ (6-2), and from these observations obtain the absolute intensity of this emission and its rotational temperature, which represents the temperature of the atmosphere between approximately 85 and $90 \mathrm{~km}$. Later, in 2005, a CCD camera replaced the Photometer and since then the mesospheric temperature has been observed. All the airglow observations in Ferraz station occurred between March and September, since in summer it is impossible to observe airglow at high latitudes because of the sunlight contamination in the airglow spectrum. Photos of the camera used for the establishment of the temperature (and intensity of the airglow emission), together with a diagram of the system is presented in Figure 3 (A). An example of the image spectrum of $\mathrm{OH}$ (6-2), obtained from this camera, is shown in Figure 3 (B). The equipment shown in Figure 3 (A) operated at EACF from 2004 to
2011, however in 2012 it was not possible to operate it due to the fact that EACF was temporarily deactivated in that year because of the fire accident that occurred on 25 February 2012. It is important pointed out that temperature measurements at the high atmosphere become relevant in the present days because of the global changes claimed by several researches.

The usage of airglow as a means of observing atmospheric waves has shown itself to be very important way to study and understand the dynamics of the high atmosphere and its correlation with the lower atmosphere and other upper phenomena (such as the plasma bubbles). These waves are mechanical oscillations in the atmospheric medium, with typical periodicity from approximately 5 minutes to hours and with a horizontal scale of tens and hundreds of kilometres. The optical instrument used to detect these waves from the ground is called "all-sky airglow imager", which is composed by an optical system with a lens called "fisheye" with a field of view of about $180^{\circ}$ (all-sky view), Airglow filters and a high sensitivity CCD sensor are important parts of an all-sky airglow imager. Observations of mesospheric gravity waves at EACF were undertaken for the first time in 2007 during a campaign of observations which occurred between March and October of that year (Bageston et al., 2009). Figure 4 shows the all-sky imager used at EACF to observe gravity waves. On the left panel it is shown the imager system together with the observation 


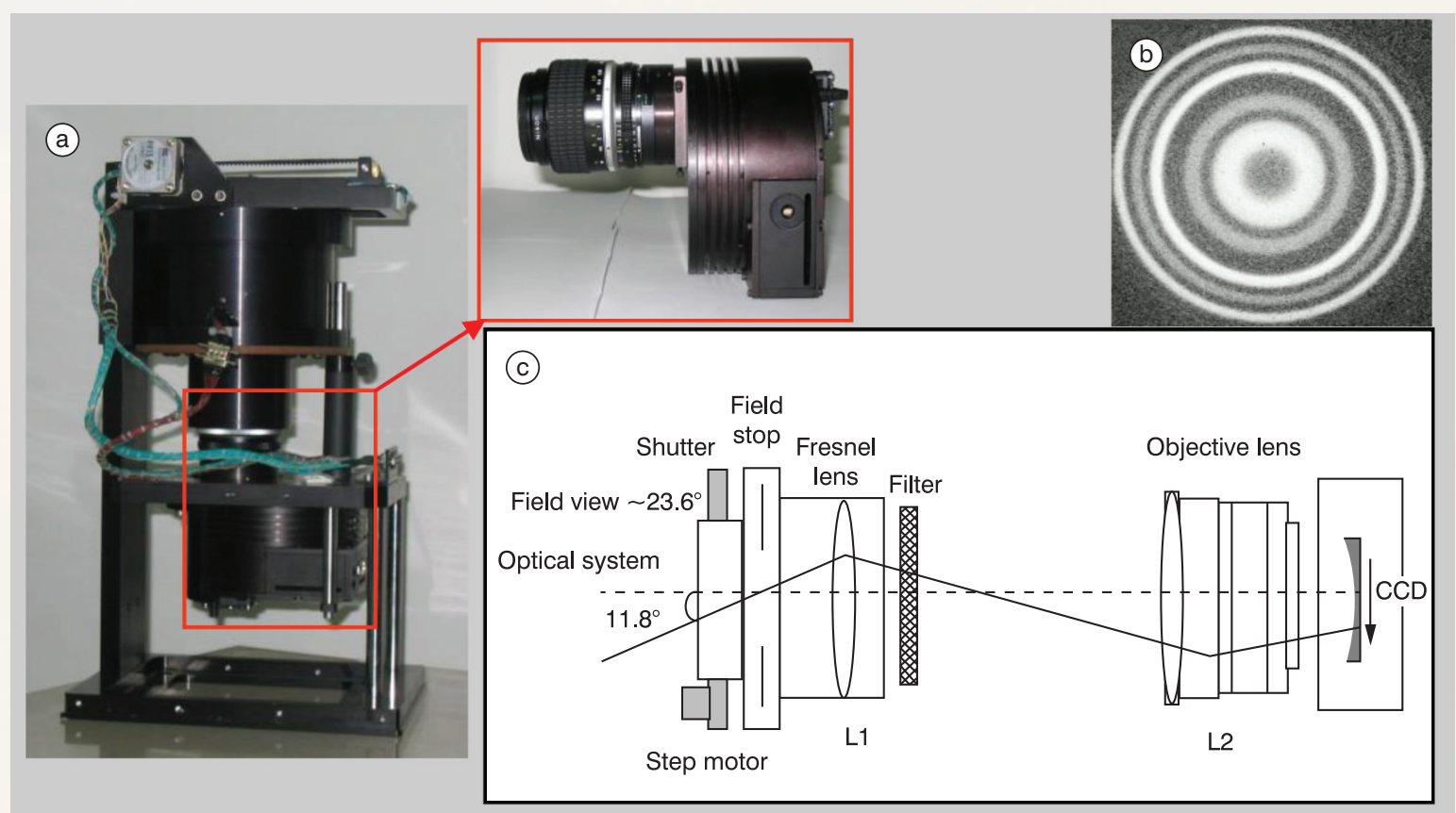

Figure 3. Spectrometer imager of atmospheric airglow (A) and the standard image (B) obtained by this system. The optical diagram of the imaging spectrometer system is shown in (C). From this type of airglow measurement it is possible to infer the temperature at around $87 \mathrm{~km}$ height.

dome and a forced air ventilation system installed to avoid condensation in the interior of the dome. On the right panel it can be seen the CCD camera in details, that is, the body of the camera and the telecentric system. The filter placed in this camera was designed to observe the near infrared airglow emission (NIR OH).

\section{Results}

From the standard images like the one showed in Figure 3 (B) it is possible to infer the temperature of the higher mesosphere (close to $90 \mathrm{~km}$ ). Each airglow image is taken at a temporal interval of approximately 1 minute in order to obtain one temperature value associated to each image. At the peak of the Antarctic winter (July-August) at EACF it is possible to obtain temperature time series with length of about 14 hours. An example of a nocturnal temporal series of temperature as obtained by the camera seen in Figure 3 is shown in Figure 5, together with data from an atmospheric model (MSIS). It is possible identify in this data a good consistency between the observed temperature and the data taken from the MSIS model, indicating that for this specific night the model was coherent with the observations. The use of the higher mesosphere temperature data together with wave observations at EACF have been undertaken recently
(Bageston et al., 2011a). However, it also planned to use these temperature data in the future to carry out climatology studies of Mesospheric temperatures over EACF.

In 2010 an airglow imager system was re-installed at EACF with the purpose of observing mesospheric gravity waves in a systematic way at that location. This system operated at EACF till 2012, which is an identical system to the one installed in 2007 (Figure 4), except for the CCD camera which was substituted due to technical issues. The small scale gravity waves (small horizontal wavelengths, $\lambda_{\mathrm{h}}$ $\sim 20-50 \mathrm{~km}$ ) detected by airglow imagers are commonly generated in the lower atmosphere by meteorological phenomena, such as cold fronts, jet stream and cyclones, or by the combination of effects of winds and the local orography (Fritts \& Alexander, 2003). Observations by means of airglow technique usually allow obtaining the horizontal wave parameters, i.e, the horizontal wavelength and phase speed, and its periodicity. Apart these parameters, also it is possible the identification of the waves propagation direction through cross-spectrum analysis. All these wave characteristics are useful as input parameters in the ray tracing modelling, which is a powerful tool used to investigate the wave sources at altitudes below or above the airglow layer where the waves are identified. Figure 6 shows 


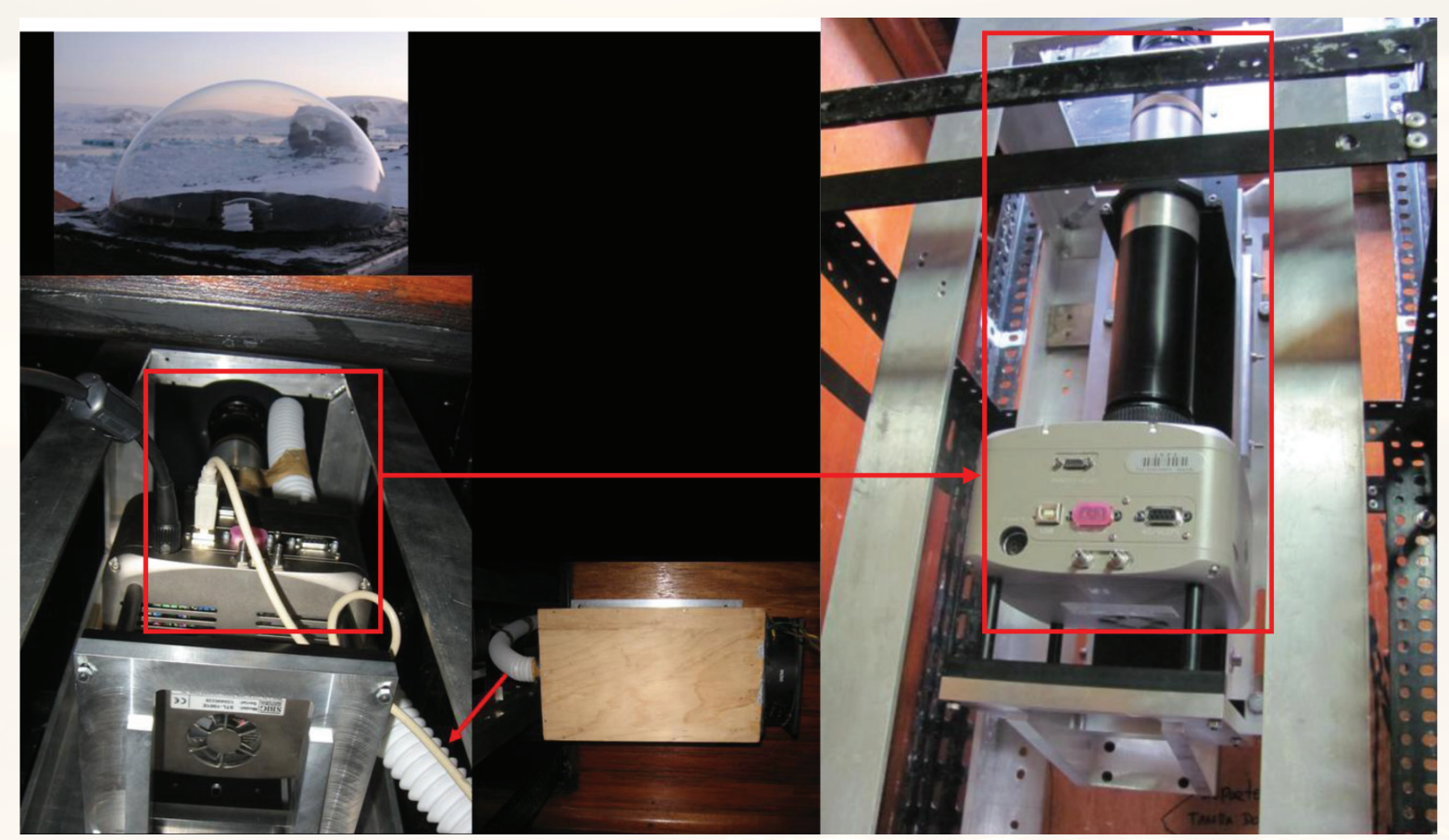

Figure 4. Airglow system for the observation of mesospheric gravity waves. In the photo on the left the camera is the exact position for observations. On the right the camera is lowered from the dome and more details of the system can be seen.

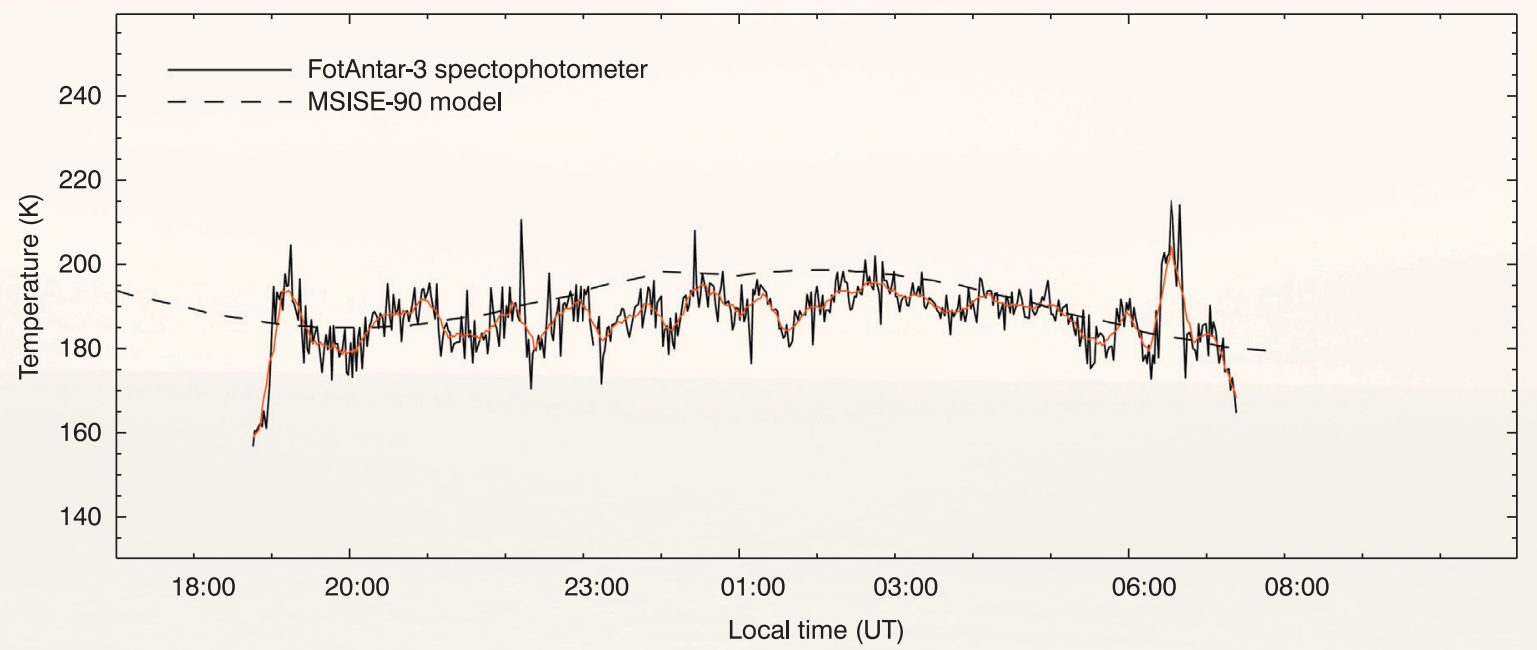

Figure 5. Example of a nocturnal temperature series (solid dark and red lines) obtained on August 052005 , compared to the MSISE-90 atmospheric model (dashed line).

an example of atmospheric gravity wave observed in 2011 above King George Island (at Ferraz station). The bright crests identified in the images represent the maximums in the airglow intensity. The airglow intensity is modulated (showing light and dark regions) due to the passage of gravity wave. In these images the stars and the Milky Way are not visible due to the usage of the temporal difference images (TD image) technique, making evident the waves in the images.

Other examples of mesospheric waves observed at Ferraz station are described in previous studies (Bageston et al., 2009, 2011a, b). In this later study it was demonstrated, 
through experimental evidence, equivalent importance of the wind and temperature in the configuration of the necessary and adequate conditions for the propagation and maintenance of mesospheric fronts in the upper atmosphere.

\section{Discussion and Conclusion}

We should emphasize that apart of the optical instruments operated at EACF, used to observe the mesosphere, a meteor radar was installed in 2010 with the aim of observe the horizontal winds in the upper mesosphere and lower thermosphere ( 80-100 km high). Beside the winds obtained by the meteor radar, this instrument has several other capabilities, such as the count of the number of meteors per hour/day, allowing studies of their variability and probable origin. This instrument also permits studies of the momentum flux variability due to gravity waves activity in the mesosphere and also is able to obtain the average daily temperatures in the meteoric region. Thus, the simultaneous operation of the meteor radar with the airglow cameras will allow the development of pioneer studies in the region of the Antarctic Peninsula. The optical observations of airglow at different sites (for example: EACF, Rothera and Tierra Del Fuego) can permit a better understanding of the dynamics of gravity waves, including its generation and propagation, in the region of the Antarctic Peninsula in the South of South America.

Regarding to the continuity of the observations and monitoring of the upper atmosphere at EACF, the installation of alternative energy sources are awaited (wind and solar) in the summer of 2013-14, or even energy generated by the provisional base installed at the beginning of 2013 enabling the continuity of research studies in next year. Furthermore, advances in collaborative relationship with other research groups that have worked at EACF is hoped for the next years, such as the groups of Meteorology, Ionosphere and ozone, apart from maintaining and expanding the existing international colaborations. The continuity of the atmospheric research activities in the Brazilian Antarctic Station, including meteorological observations, the monitoring of the ultraviolent radiation and ozone layer, besides the monitoring of the upper atmosphere is essential in order for Brazil to maintain an active role in the atmospheric research scenario in Antarctica.

\section{Acknowledgments}

This work integrates the National Institute of Science and Technology Antarctic Environmental Research (INCTAPA) that receives scientific and financial support from the National Council for Research and Development (CNPq process: $n^{\circ}$ 574018/2008-5) and Carlos Chagas Research Support Foundation of the State of Rio de Janeiro (FAPERJ $\left.\mathrm{n}^{\circ} \mathrm{E}-16 / 170.023 / 2008\right)$. The authors also acknowledge the support of the Brazilian Ministries of Science, Technology and Innovation (MCTI), of Environment (MMA) and InterMinistry Commission for Sea Resources (CIRM).

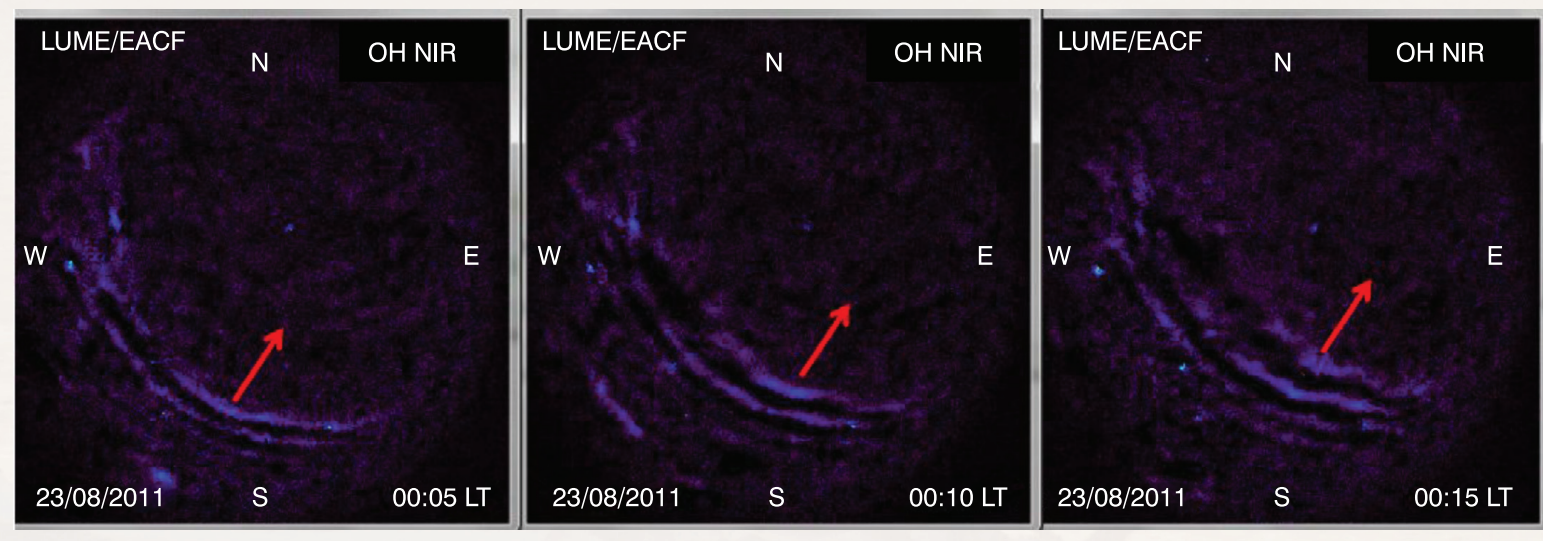

Figure 6. An example of a mesospheric gravity wave identified through the airglow emission in the near-infrared band of the hydroxyl (OH NIR). This wave event was observed on the night of 22 to 23 August 2011. The arrows drawn on the images indicate the wave propagation direction. 


\section{References}

Amorim, D.C.M.; Pimenta, A.A.; Bittencourt, J. A. \& Fagundes P. R. (2011). Long-term study of medium-scale traveling ionospheric disturbances using O I 630 nm all-sky imaging and ionosonde over Brazilian low latitudes. Geophysical Research Letters, 116(s/n): A06312. http://dx.doi.org/10.1029/2010JA016090

Bageston, J. V.; Gobbi, D.; Takahashi, H. \& Wrasse, C. M. (2007). Development of Airglow OH Temperature Imager for Mesopheric Study. Brazilian Journal of Geophysics, 25(2): 27-34. Available from: http://www.scielo.br/pdf/rbg/v25s2/ a04v25s2.pdf.

Bageston, J. V.; Wrasse, C. M.; Gobbi, D. Tahakashi, H. \& Souza, P. B. (2009). Observation of Mesospheric Gravity Waves at Estação Antártica Comandante Ferraz (62S), Antarctica. Annales Geophysicae, 27(s/n): 2593-2598. http://dx.doi. org/10.5194/angeo-27-2593-2009

Bageston, J. V.; Wrasse, C. M., Hibbins; R. E, Batista; P. P., Gobbi, D.; Takahashi, H.; Fritts, D. C.; Andrioli, V. F.; Fechine, J. \& Denardini, C. M. (2011a). Case Study of a Mesospheric Wall Event over Ferraz Station, Antarctica (62 $\mathrm{S})$. Annales Geophysicae, 29(s/n): 209-219. http://dx.doi.org/10.5194/angeo-29-209-2011

Bageston, J. V.; Wrasse, C. M.; Batista, P. P.; Hibbins R. E.; , Fritts, D. C.; Gobbi, D. \& Andrioli, V. F. (2011b). Observation of a mesospheric front in a thermal-doppler duct over King George Island, Antarctica. Atmospheric Chemistry and Physics, 11(s/n): 12137-12147. http://dx.doi.org/10.5194/acp-11-12137-2011

Espy, P., Jones, G., Swenson, G., Tang, J. \& Taylor, M. J (2004). Seasonal variations of gravity wave momentum flux in the Antarctic mesosphere and lower thermosphere. Geophysical Research Letters, 109(s/n): 1-9. http://dx.doi. org/10.1029/2003JD004446

Fritts, D. C.; Janches, D.; limura, H.; Hocking, W. K.; Bageston, J. V.; Leme, N. M. P. (2012). Drake Antarctic Agile Meteor Radar first results: Configuration and comparison of mean and tidal wind and gravity wave momentum flux measurements with Southern Argentina Agile Meteor Radar. Journal of Geophysical Research, 117(s/n): D02105. http://dx.doi. org/10.1029/2011JD016651

Fritts, D.C. \& Alexander, M.J. (2003). Gravity wave dynamics and effects in the middle atmosphere. Journal of Geophysical Research, 41(s/n): 1--46.

Medeiros, A.F.; Takahashi, H.; Buriti, R.A.; Fechine, J.; Wrasse, C. M. \& Gobbi D. (2007). MLT gravity wave climatology in the South America equatorial region observed by airglow imager. Annales Geophysicae, 25(s/n): 399-406. http://dx.doi. org/10.5194/angeo-25-399-2007

Nielsen, K.; Taylor, M.J.; Stockwell, R.G. \& Jarvis, M.J. (2006). An unusual mesospheric bore event observed at high latitudes over Antarctica. Geophysical Research Letters, 33(L07803): 1-4. http://dx.doi.org/10.1029/2005GL025649

Nielsen, K.; Taylor, M.; Hibbins, R. \& Jarvis, M. (2009). Climatology of short-period mesospheric gravity waves over Halley, Antarctica $\left(76^{\circ} \mathrm{S}, 27^{\circ} \mathrm{W}\right)$. Journal of Atmospheric and Solar-Terrestrial Physics, 71(s/n): 991-1000. http://dx.doi.org/10.1016/j. jastp.2009.04.005

Paulino, I.; Medeiros, A.F.; Buriti, R.A.; Takahashi, H.; Sobral, J.H.A \& Gobbi, D. (2011). Plasma bubble zonal drift characteristics observed by airglow images over Brazilian tropical region. Brazilian Journal of Geophysics, 29(2): 239-246. http://dx. doi. org/10.1590/S0102-261X2011000200003

Smith, S.M.; Taylor, M.J.; Swenson, G.R.; She, C.; Hocking, W.; Baumgardner, J. \& Mendillo, M.A. (2003). Multidiagnostic investigation of the mesospheric bore phenomenon. Journal of Geophysical Research, 108(s/n): 13-20.

Taylor, M.J.; Turnbull, D.N. \& Lowe, R.P. (1995). Spectrometric and imaging measurements of a spectacular gravity wave event observed during the ALOHA-93 campaign. Geophysical Research Letters, 20 (s/n): 2849-2852.

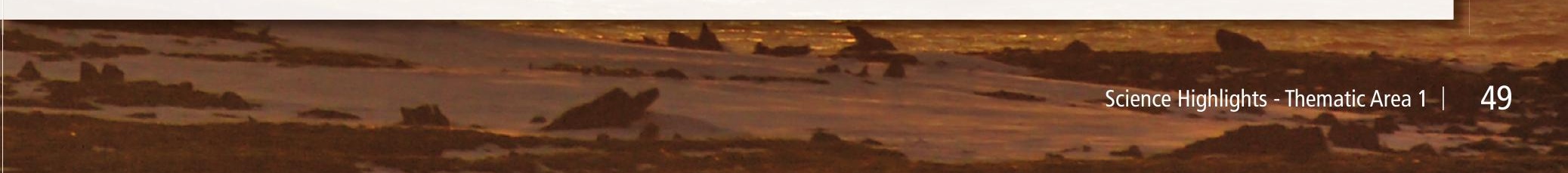

